\title{
Hypocholesterolemic Effects of Mevinolin in Patients With Heterozygous Familial Hypercholesterolemia
}

\section{Roger Illingworth and Gary J. Sexton}

Division of Endocrinology, Metabolism, and Clinical Nutrition, Department of Medicine, and Department of Biochemistry, The Oregon Health Sciences University, Portland, Oregon 97201

bstract. We have evaluated the hypolipidemic effects of mevinolin, a competitive inhibitor of 3-hydroxy3-methylglutaryl coenzyme A reductase, the rate-limiting enzyme in cholesterol biosynthesis in 13 patients with heterozygous familial hypercholesterolemia (FH). Patients were maintained on a low-cholesterol diet and received sequentially increasing doses of $5,10,20$, and $40 \mathrm{mg}$ of mevinolin twice daily for a period of $1 \mathrm{mo}$ on each dose. Plasma concentrations of low density lipoprotein cholesterol decreased by $19.8 \%$ on the $5 \mathrm{mg}$ twice daily dose ( $P<0.05$ vs. base line), $28.4 \%$ on 10 $\mathrm{mg}$ of mevinolin twice daily $(P<0.05$ vs. $5 \mathrm{mg}$ twice daily), $35 \%$ on $20 \mathrm{mg}$ of mevinolin twice daily $(P$ $<0.05$ vs. $10 \mathrm{mg}$ twice daily), and $37.7 \%$ on $40 \mathrm{mg}$ of mevinolin twice daily (not statistically different from 20 $\mathrm{mg}$ twice daily). Concentrations of high density lipoprotein cholesterol remained stable on all doses of mevinolin whereas plasma triglyceride levels fell significantly on the $20 \mathrm{mg}(-30.7 \%)$ and $40 \mathrm{mg}(-34.3 \%)$ twice daily doses of mevinolin. Mevinolin was well tolerated and all patients completed the study period. Side effects during the period of study were limited to transient insomnia and headaches in two patients, transient increases in alkaline phosphatase in three patients, and a modest but sustained increase in alkaline phosphatase in a fourth patient. These results indicate that mevinolin

This work was presented in part at the 56th Scientific Meeting of the American Heart Association, Anaheim, CA, 1983, and was published in abstract (1983. Circulation. 68:111-188).

Address correspondence to Dr. Illingworth, The Oregon Health Sciences University, Portland, OR 97201.

Received for publication 13 February 1984 and in revised form 16 August 1984.

J. Clin. Invest.

(c) The American Society for Clinical Investigation, Inc.

$0021-9738 / 84 / 12 / 1972 / 07 \quad \$ 1.00$

Volume 74, December 1984, 1972-1978 is an effective hypolipidemic agent in patients with heterozygous $\mathrm{FH}$ but that the optimal doses in these patients are greater than those previously reported in normal volunteers. If long-term safety can be satisfactorily established, mevinolin offers considerable promise in the therapy of heterozygous FH.

\section{Introduction}

Patients with heterozygous familial hypercholesterolemia $(\mathrm{FH})^{1}$ constitute a group at high risk for the premature development of atherosclerosis (1-5). This disorder, which is inherited as an autosomal dominant trait (6), is biochemically heterogeneous and results from abnormalities in receptor-mediated uptake and metabolism of low density lipoprotein (LDL) by cells (7). Kinetic studies of ${ }^{125}$ I-labeled LDL in patients with heterozygous FH have indicated that the reduced number of high affinity LDL receptors expressed on their cells results in a reduced fractional rate of catabolism of LDL from plasma (8-10) and is associated with lifelong hypercholesterolemia.

Because of their strong predilection for premature coronary artery disease, patients with heterozygous FH constitute a population in whom long-term hypocholesterolemic therapy to reduce elevated levels of LDL cholesterol is most clearly indicated. Current therapy of this disorder consists of dietary restriction of saturated fats and cholesterol and the use of pharmaceutical agents (11-14), or less commonly, surgical bypass of the distal ileum (15). The bile acid sequestrants, cholestyramine or colestipol, or nicotinic acid, are considered the agents of choice for single drug therapy (16-18). The former agents, like distal ileal bypass (19), increase fecal bile acid excretion and stimulate compensatory increases in the hepatic conversion of cholesterol to bile acids. This in turn promotes further compensatory increases in de novo cholesterol biosynthesis and in the number of high affinity LDL receptors present on hepatocyte membranes (20); the latter increase

1. Abbreviations used in this paper: CPK, creatine phosphokinase; FH, familial hypercholesterolemia; HMG-COA, 3-hydroxy-3-methylglutaryl coenzyme A. 
results in an enhanced rate of receptor-mediated LDL catabolism (21) and a fall in the concentrations of this lipoprotein in plasma.

The recent development of two specific competitive inhibitors of the rate-limiting enzyme in cholesterol biosynthesis, 3hydroxy-3-methylglutaryl coenzyme A (HMG-COA) reductase, compactin (21-25) and mevinolin (26-28), has provided a potential new avenue of therapy for patients with primary hypercholesterolemia. Recent studies (29) indicate that the hypocholesterolemic effects of mevinolin result mainly from an increased fractional rate of catabolism of LDL (mediated by an increase in the number of high affinity LDL receptors), but that the drug may concurrently reduce LDL synthesis. In a study to assess the dose-response relationships with mevinolin in normal male volunteers, Tobert et al. (28) noted reductions of $35-45 \%$ in the plasma concentrations of LDL cholesterol when the subjects received doses of $6.25-50.0 \mathrm{mg}$ of mevinolin twice daily. Although the mean reduction in LDL cholesterol levels in subjects receiving $50 \mathrm{mg}$ of mevinolin twice daily was slightly lower than that observed in subjects receiving 6.25 , 12.5 , or $25 \mathrm{mg}$ twice daily, this difference was not statistically significant. In the present study we have examined the doseresponse relationships between changes in the concentrations of plasma lipids and lipoproteins and mevinolin dosage in adult patients with well-characterized heterozygous $\mathbf{F H}$.

\section{Methods}

Subjects. 13 adult patients with severe type II hypercholesterolemia, in whom prior hypolipidemic therapy (11-14) had failed to lower serum cholesterol values below $300 \mathrm{mg} / \mathrm{dl}$, participated in the study (Table I). Patients were diagnosed to have heterozygous FH on the basis of persistent primary hypercholesterolemia $>350 \mathrm{mg} / \mathrm{dl}$, tendon xanthomas, an inheritance pattern consistent with autosomal dominant, and primary hypercholesterolemia in other family members with an absence of multiple phenotypes. Incomplete family data were available for one patient (case 5), and in this case, the diagnosis of heterozygous FH was based on the presence of tendon xanthomas, long-standing primary hypercholesterolemia, and a reduced rate of high affinity degradation of ${ }^{125}$ I-labeled LDL by freshly isolated mononuclear cells (35\% of the values in normal controls). All patients had normal thyroid, renal, and hepatic function and none had diabetes or were taking exogenous estrogens. Three patients (cases 1, 5, and 6) were receiving therapy for hypertension with a thiazide diuretic and propanolol, and two others (cases 3 and 12) were taking propanolol or atenolol for symptoms of angina pectoris. In all cases, this therapy was continued unaltered during the period of treatment with mevinolin. The response of these patients did not differ from those not on antihypertensive therapy. None of the other eight patients was receiving drugs known to affect lipid metabolism.

Study protocol. All patients were seen on an outpatient basis in the Clinical Research Center or Lipid Disorders Clinic of The Oregon Health Sciences University. Informed consent was obtained from each patient and the protocol was approved by the Human Research Committee of this institution. Hypolipidemic drugs were withdrawn for a minimum period of 3 wk before blood samples were obtained for initial base-line lipid values. Two base-line values were obtained in each patient. No patient had previously been treated with probucol. Before therapy with mevinolin all patients underwent a complete history and physical examination, and blood samples were obtained for lipid and lipoprotein analysis, hematologic studies, and clinical chemistry profiles. Dietary compliance was assessed by $24-\mathrm{h}$ recalls. All patients were habituated to a low-cholesterol $(<300 \mathrm{mg} / \mathrm{d})$ diet

Table I. Clinical Characteristics and Lipoprotein Profiles of Patients with Heterozygous Familial Hypercholesterolemia

\begin{tabular}{|c|c|c|c|c|c|c|c|c|c|}
\hline \multirow[b]{2}{*}{ Patient no. } & \multirow{2}{*}{$\begin{array}{l}\text { Age } \\
(y r) / \operatorname{sex}\end{array}$} & \multirow[b]{2}{*}{ Weight } & \multirow{2}{*}{$\begin{array}{l}\text { Tendon } \\
\text { xanthoma }\end{array}$} & \multirow{2}{*}{$\begin{array}{l}\text { Corneal } \\
\text { arcus }\end{array}$} & \multicolumn{3}{|c|}{ Plasma cholesterol } & \multirow{2}{*}{$\begin{array}{l}\text { Plasma } \\
\text { triglyc- } \\
\text { erides }\end{array}$} & \multirow[b]{2}{*}{ Previous drug therapy } \\
\hline & & & & & Total & LDL & HDL & & \\
\hline & & $k g$ & & & $m g / d l$ & & & $m g / d l$ & \\
\hline 1 & $68 \mathrm{~F}$ & 80 & + & + & 447 & 364 & 54 & 149 & col, nic, pb, clo \\
\hline 2 & $58 \mathrm{~F}$ & 58 & + & + & 464 & 356 & 72 & 184 & col, nic, clo \\
\hline 3 & $56 \mathrm{~F}$ & 57 & + & + & 493 & 407 & 64 & 116 & col, nic, pb \\
\hline 4 & $55 \mathrm{M}$ & 74 & + & + & 457 & 389 & 54 & 125 & col, nic \\
\hline 5 & $51 \mathrm{M}$ & 79 & + & - & 488 & 400 & 37 & 265 & col, nic, neo, clo, ct \\
\hline 6 & $49 \mathrm{M}$ & 94 & + & + & 530 & 441 & 50 & 193 & col, nic, neo, pb, clo \\
\hline 7 & $44 \mathrm{~F}$ & 57 & + & + & 404 & 331 & 59 & 68 & col, nic, clo, ct \\
\hline 8 & $42 \mathrm{~F}$ & 55 & + & - & 395 & 323 & 54 & 89 & col, nic \\
\hline 9 & $38 \mathrm{~F}$ & 55 & + & + & 401 & 329 & 41 & 157 & col, nic, pb \\
\hline 10 & $36 \mathrm{~F}$ & 78 & + & + & 446 & 366 & 44 & 184 & col, nic, pb, clo \\
\hline 11 & $35 \mathrm{M}$ & 68 & + & - & 363 & 285 & 26 & 262 & col, nic \\
\hline 12 & $32 \mathrm{M}$ & 73 & + & + & 503 & 421 & 28 & 252 & col, nic, clo \\
\hline 13 & $31 \mathrm{~F}$ & 68 & + & + & 465 & 390 & 60 & 71 & col, nic \\
\hline Mean \pm SEM & $46 \pm 3$ & $69 \pm 3$ & 13 & 10 & $450 \pm 14$ & $369 \pm 12$ & $49 \pm 4$ & $163 \pm 19$ & \\
\hline
\end{tabular}

Abbreviations used in this table: col, colestipol; nic, nicotinic acid; pb, probucol; neo, neomycin; clo, clofibrate; ct, cholestyramine. 
containing $<10 \%$ of calories as saturated fat, and their overall dietary intakes and body weights remained stable during the period of therapy (mean+SEM, $69.0 \pm 3.4 \mathrm{~kg}$ at base line, $69.1 \pm 3.6 \mathrm{~kg}$ on $5 \mathrm{mg}$ of mevinolin twice daily, $69.3 \pm 3.5 \mathrm{~kg}$ on $10 \mathrm{mg}$ twice daily, $69.3 \pm 3.6 \mathrm{~kg}$ on $20 \mathrm{mg}$ twice daily, and $69.1 \pm 3.5 \mathrm{~kg}$ on $40 \mathrm{mg}$ of mevinolin twice daily). After the 4-wk base-line period (during which lipid values remained stable), patients were begun on single drug therapy with mevinolin at a dose of $5 \mathrm{mg}$ twice daily (7:00-8:00 a.m. and 6:008:00 p.m.). Patients were seen at intervals of $2 \mathrm{wk}$ at which time inquiries were made as to any side effects, brief physical examinations were performed, and blood samples were taken for lipid and lipoprotein analysis and clinical chemistry profiles.

In initial studies five patients were sequentially maintained on doses of 5,10 , or $20 \mathrm{mg}$ mevinolin twice daily for a period of 6 wk on each dose. Plasma cholesterol values in these patients were as follows: base line, $444 \pm 21 \mathrm{mg} / \mathrm{dl}$, (mean $\pm \mathrm{SEM}$ ); $5 \mathrm{mg}$ mevinolin twice daily, $392 \pm 20 \mathrm{mg} / \mathrm{dl}$ after $2 \mathrm{wk}, 363 \pm 21 \mathrm{mg} / \mathrm{dl}$ after $4 \mathrm{wk}, 355 \pm 16$ $\mathrm{mg} / \mathrm{dl}$ after $6 \mathrm{wk} ; 10 \mathrm{mg}$ mevinolin twice daily, $336 \pm 20 \mathrm{mg} / \mathrm{dl}$ after 2 wk, $325 \pm 16 \mathrm{mg} / \mathrm{dl}$ after $4 \mathrm{wk}$, and $324 \pm 14 \mathrm{mg} / \mathrm{dl}$ after $6 \mathrm{wk}$; and on $20 \mathrm{mg}$ of mevinolin twice daily, $317 \pm 14 \mathrm{mg} / \mathrm{dl}$ after $2 \mathrm{wk}, 296 \pm 17$ $\mathrm{mg} / \mathrm{dl}$ after $4 \mathrm{wk}$, and 309 $\pm 14 \mathrm{mg} / \mathrm{dl}$ after 6 wk. Statistical analysis (one-way analysis of variance with repeated measures) indicated a significant $(P<0.05)$ further decrease in total cholesterol between wk 2 and 4 on the $5 \mathrm{mg}$ twice daily dosage of mevinolin but no statistical significance between the values at 4 and 6 wk. For all other mevinolin doses (10,20, and $40 \mathrm{mg}$ twice daily) the lipid values after 2 wk on each dose were not statistically different from those at 4 and 6 wk. This analysis also indicates that the statistically significant changes in total and LDL cholesterol that occurred in response to increasing doses of mevinolin (up to $20 \mathrm{mg}$ twice daily) are attributable to the higher dosage rather than a carryover effect from previous administration of the lower dose. Based on these preliminary studies, which indicated that stable lipid values are attained in 4 wk or less on a given dose of mevinolin, changes to a higher dose were made after $4 \mathrm{wk}$ on each dose of mevinolin in the remainder of the study patients. The data reported in this paper therefore represent values obtained after a 4-wk period on each dose of mevinolin. Blood samples for hematologic (white blood cell count, hemoglobin, hematocrit, and platelet count) and clinical chemistry determinations (glucose, blood urea nitrogen, creatinine, electrolytes, uric acid, bilirubin, lactic dehydrogenase, alkaline phosphatase, serum glutamic oxaloacetic transaminase, and creatine phosphokinase (CPK)) were obtained at 4-wk intervals. Compliance (assessed by verbal questions and by pill counts) was consistently $>95 \%$ in this selected and highly motivated patient population.

Analytical methods. Blood samples for lipid and lipoprotein analyses were obtained after an overnight fast into tubes containing $1 \mathrm{mg} / \mathrm{dl}$ EDTA as anticoagulant. Plasma was separated at $4^{\circ} \mathrm{C}$ in a refrigerated centrifuge and total cholesterol and triglycerides measured on an autoanalyzer II (30). High density lipoprotein (HDL) cholesterol was determined on the supernatant after heparin manganese precipitation of plasma. The cholesterol content of the HDL fraction was determined directly and the LDL concentration was calculated (30). Lipid determinations were standardized with samples of known composition obtained from the Center for Disease Control, Atlanta, GA. Clinical chemistry and hematologic determinations were performed using routine automated methods in the Clinical Pathology laboratory, The Oregon Health Sciences University. Statistical analyses were performed by oneway analysis of variance with repeated measures. The Neuman-Keuls procedure was used for post hoc comparisons (31).

\section{Results}

Changes in the concentrations of plasma total and LDL cholesterol in the 13 patients with heterozygous FH during sequential therapy with increasing doses of mevinolin are shown in Fig. 1 and 2 . In each figure the results represent single-point determinations on each patient after $4 \mathrm{wk}$ on a given dose of mevinolin. Total plasma cholesterol concentrations fell from a base-line value of $450 \pm 14$ (mean \pm SEM) to $373 \pm 13 \mathrm{mg} / \mathrm{dl}$ after $4 \mathrm{wk}$ on $5 \mathrm{mg}$ twice daily of mevinolin ( $P$ $<0.05), 344 \pm 15 \mathrm{mg} / \mathrm{dl}$ on $10 \mathrm{mg}$ twice daily $(P<0.05$ vs. the $5 \mathrm{mg}$ twice daily dose), $313 \pm 15 \mathrm{mg} / \mathrm{dl}$ on $20 \mathrm{mg}$ of mevinolin twice daily $(P<0.05$ vs. the $10 \mathrm{mg}$ twice daily dose), and $300 \pm 15 \mathrm{mg} / \mathrm{dl}$ after $4 \mathrm{wk}$ on $40 \mathrm{mg}$ twice daily of mevinolin. The latter two values are not significantly different. Changes in the concentrations of LDL cholesterol in the 13 patients during sequential therapy with mevinolin paralleled those for total cholesterol (Fig. 2). Concentrations of LDL cholesterol decreased from a base-line value of $369 \pm 12$ (mean \pm SEM) to $296 \pm 11 \mathrm{mg} / \mathrm{dl}$ after 4 wk on $5 \mathrm{mg}$ of mevinolin twice daily $(P<0.05$ vs. base line), $264 \pm 14 \mathrm{mg} / \mathrm{dl}$ on $10 \mathrm{mg}$ twice daily of mevinolin $(P<0.05 \mathrm{vs} .5 \mathrm{mg}$ twice daily), $240 \pm 14 \mathrm{mg} / \mathrm{dl}$ on $20 \mathrm{mg}$ of mevinolin twice daily $(P$ $<0.05$ vs. $10 \mathrm{mg}$ twice daily dose), and $230 \pm 13 \mathrm{mg} / \mathrm{dl}$ on 40 $\mathrm{mg}$ twice daily of mevinolin (not significantly different from the $20 \mathrm{mg}$ twice daily dosage). These changes correspond to reductions of $19.8,28.4,35$, and $37.7 \%$ in the concentrations of LDL cholesterol on the $5,10,20$, and $40 \mathrm{mg}$ twice daily doses of mevinolin, respectively (Table II). There was no significant correlation between the initial concentrations of total or LDL cholesterol and subsequent changes in response to therapy with mevinolin $(r=-0.36, P=0.22)$.

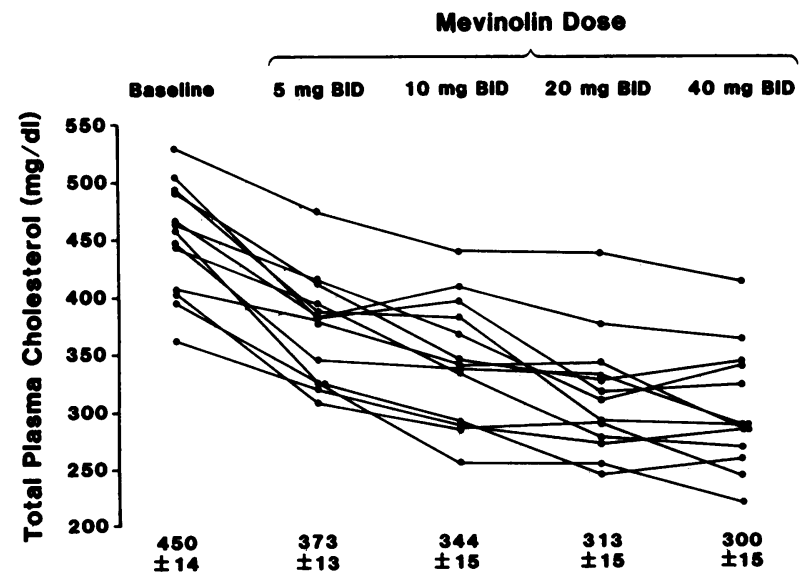

Figure 1. Changes in the plasma concentrations of total cholesterol during sequential therapy with increasing doses of mevinolin in patients with heterozygous familial hypercholesterolemia. Each point represents the value obtained after 4 wk on each dose of mevinolin in each patient. 


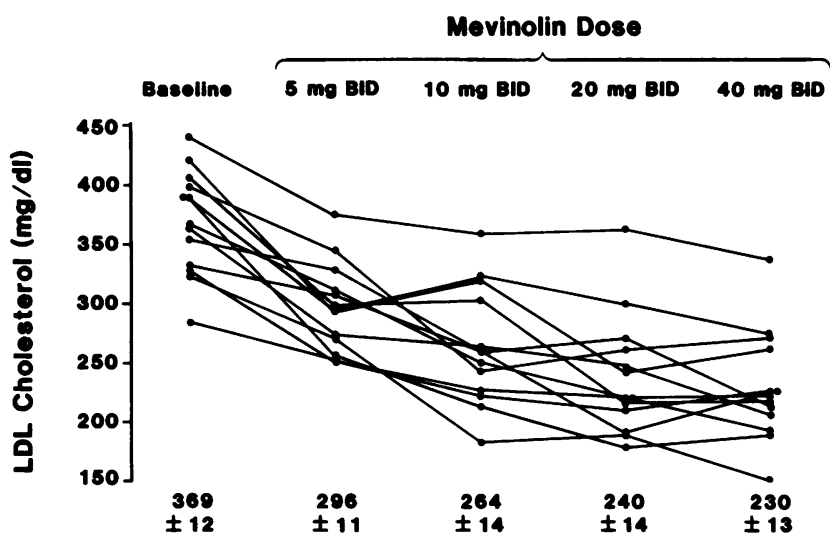

Figure 2. Changes in the plasma concentrations of LDL cholesterol during sequential therapy with increasing doses of mevinolin in patients with heterozygous familial hypercholesterolemia. Each point represents the value obtained after $4 \mathrm{wk}$ on each dose of mevinolin in each patient.

Although the individual responses show considerable variation, the overall trend was for a progressive decrease in the concentrations of both total and LDL cholesterol at doses of mevinolin up to $20 \mathrm{mg}$ twice daily, at which point a plateau was reached (Fig. 3). As illustrated in Fig. 3 and Table II, plasma triglycerides fell slightly on the lower doses of mevinolin (a 6\% decrease on the $5 \mathrm{mg}$ twice daily dosage and 11\% fall on the $10 \mathrm{mg}$ twice daily regimen) but were significantly lower on the 20 or $40 \mathrm{mg}$ twice daily regimens. Plasma triglyceride concentrations on the latter doses of mevinolin decreased by 30.7 and $34.3 \%$, respectively ( $P<0.05$ vs. base line). Concentrations of HDL cholesterol remained stable throughout the period of study and were not influenced by mevinolin (Fig. 3, Table II).

Table II. Percentage Changes in the Plasma Concentrations of Lipids and Lipoproteins During Sequential Therapy with Increasing Doses of Mevinolin in 13 Patients with Heterozygous Familial Hypercholesterolemia

\begin{tabular}{lcccc}
\hline \multicolumn{5}{c}{ Mevinolin dose $(m g)$} \\
\cline { 2 - 5 } & 5 & 10 & 20 & 40 \\
\hline Total cholesterol & $-17.1^{*} \ddagger$ & $-23.5^{*} \ddagger$ & $-30.4^{*} \ddagger$ & $-33.3 \ddagger$ \\
LDL cholesterol & $-19.8^{*} \ddagger$ & $-28.4^{*} \ddagger$ & $-35.0^{*} \ddagger$ & $-37.7 \ddagger$ \\
HDL cholesterol & -8.1 & +2.0 & 0 & +2.0 \\
Triglycerides & -6.1 & -11.0 & $-30.7 \ddagger$ & $-34.3 \ddagger$
\end{tabular}

The data are derived from the lipid values obtained after $4 \mathrm{wk}$ on each dose of mevinolin. Each dose was given twice daily.

* Significantly different from previous dose (or base line for $5 \mathrm{mg}$ twice daily).

‡ Significantly different from base line.

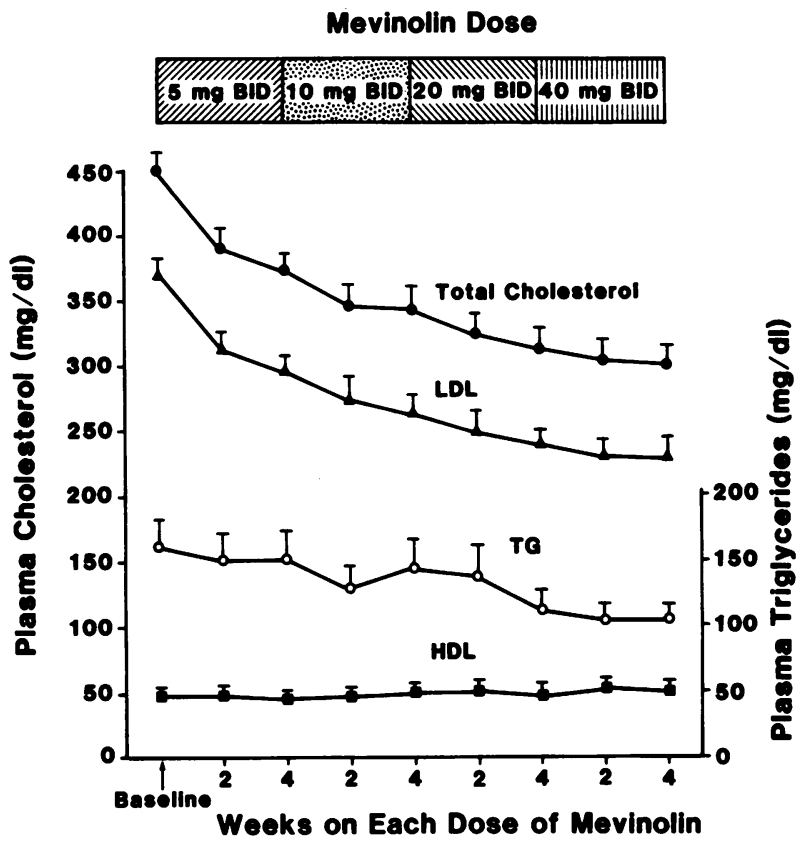

Figure 3. Changes in the concentrations of plasma total, LDL, and HDL cholesterol, and triglycerides (TG) in 13 patients with heterozygous familial hypercholesterolemia during sequential therapy with increasing doses of mevinolin. Each point represents the mean \pm SEM from single determinations on each patient at each time point.

Mevinolin was uniformly well tolerated and no patient was withdrawn from the study. Two patients reported subjective but transient changes while taking mevinolin. These were an increased frequency of headaches in one patient (case 7) and insomnia in the second (case 9), both at a time when they were receiving $40 \mathrm{mg}$ of mevinolin twice daily. In both cases these symptoms have resolved and the patients remain on therapy ( $20 \mathrm{mg}$ twice daily). Biochemical changes have been limited to mild and transient rises in alkaline phosphatase (from 80-110 units/liter before therapy to 120-140 units/liter; normal, 35-105 units/liter) in three patients (cases 1, 2, and 11); the first two patients were receiving $20 \mathrm{mg}$ mevinolin twice daily and the third, $5 \mathrm{mg}$ twice daily. In all three cases the values returned to base line with continued therapy. A modest but more sustained increase in alkaline phosphatase was noted in a fourth patient (case 12) in whom alkaline phosphatase levels increased from a base-line level of 95 units/ liter (normal, 35-105 units/liter) to 121 units/liter on $10 \mathrm{mg}$ mevinolin twice daily, 134 units/liter on $20 \mathrm{mg}$ mevinolin twice daily, and 135 units/liter on $40 \mathrm{mg}$ mevinolin twice daily. Serum glutamic oxaloacetic transaminase and bilirubin remained within the normal range. This patient was on concurrent therapy with diltiazam, and because of the previous refractory nature of his hypercholesterolemia, is being maintained on combined therapy with $20 \mathrm{mg}$ of mevinolin twice daily and $10 \mathrm{~g}$ colestipol twice daily. Serum cholesterol levels 
on this combined regimen have decreased from an initial value of 503 to $268 \mathrm{mg} / \mathrm{dl}$ on combined drug therapy. Alkaline phosphatase after 8 and 11 mo of continuous mevinolin therapy (the last 6 mo at a dose of $20 \mathrm{mg}$ twice daily) have been 121 and 99 units/liter; other liver function tests remain normal.

\section{Discussion}

The hypocholesterolemic effects of two specific competitive inhibitors of the rate-limiting enzyme in cholesterol biosynthesis HMG-COA reductase, compactin (23-25) and mevinolin (2729), have been reported in normal subjects and in patients with heterozygous $\mathrm{FH}$, but the dose-response relationships have only been examined in the former (28). In a multicenter trial, Tobert et al. (28) examined the hypolipidemic effects of mevinolin given in doses of $6.25,12.5,25$, and $50 \mathrm{mg}$ twice daily to normal male volunteers. Concentrations of LDL cholesterol fell by $35-45 \%$ in all groups and despite a slightly larger decrease on the $50 \mathrm{mg}$ twice daily dosage, differences between groups were not significant. These results in normal subjects differ from those of the present study in patients with heterozygous FH in whom significantly greater decreases in the concentrations of total and LDL cholesterol were found on the 5,10 , and $20 \mathrm{mg}$ twice daily doses of mevinolin. Concentrations of LDL cholesterol were reduced by $35 \%$ on $20 \mathrm{mg}$ of mevinolin twice daily, at which dose a plateau effect was observed, and increases in mevinolin dose above this (to $40 \mathrm{mg}$ twice daily) resulted in insignificant further changes (mean $37.7 \%$ below base line).

The overall magnitude of lipid lowering reported in the present study with optimal doses of mevinolin is slightly greater than noted in previous studies in which fixed doses of mevinolin or compactin have been administered to patients with heterozygous FH. Mabuchi et al. (24) noted a 29\% decrease in LDL cholesterol concentrations in seven Japanese patients with heterozygous FH who were treated with 30-60 $\mathrm{mg} /$ day of compactin. Similar changes were recently reported by Bilheimer et al. (29) in six patients with heterozygous FH who received $20 \mathrm{mg}$ of mevinolin twice daily for a 6-wk period and in whom the concentrations of LDL cholesterol fell by $27 \%$.

No significant changes were observed in the concentrations of HDL cholesterol during therapy with mevinolin in doses up to $\mathbf{4 0} \mathrm{mg}$ twice daily. These findings are similar to those reported by Mabuchi et al. (24) in patients with heterozygous FH treated with compactin or very recently with a combination of compactin plus cholestyramine (25).

The precise mechanism(s) responsible for the hypolipidemic effects of mevinolin in patients with heterozygous FH remains to be fully elucidated. In dogs, mevinolin in a dose of $10 \mathrm{mg} /$ $\mathrm{kg}$ per day has been shown to cause a small increase in the fractional catabolic rate of ${ }^{125} \mathrm{I}-\mathrm{LDL}$, but the primary hypolipidemic effect was mediated by a $50 \%$ reduction in the rate of
LDL synthesis (20). These findings contrast with those of a recent study (29) in which the kinetics of ${ }^{125} \mathrm{I}-\mathrm{LDL}$ were examined in six patients with heterozygous FH on diet only and diet plus mevinolin ( $20 \mathrm{mg}$ twice daily). In this report the fractional catabolic rate of LDL apoprotein B increased from a mean of 0.30 pools/d on diet only to 0.41 pools $/ \mathrm{d}$ on mevinolin. Although the mean calculated rate of synthesis of LDL apoprotein B did not change significantly $(19.2 \mathrm{mg} / \mathrm{kg}$ per day on diet only vs. $18.4 \mathrm{mg} / \mathrm{kg}$ per day on mevinolin), in the patients as a whole it fell modestly in five of the six individual subjects but increased appreciably in the sixth. These results indicate that the observed decrease in LDL cholesterol levels in patients with heterozygous FH treated with $20 \mathrm{mg}$ of mevinolin twice daily is mediated primarily by an increased fractional rate of LDL catabolism, but that some reduction in LDL synthesis may occur concurrently. Indirect support for an inhibitory effect of mevinolin on LDL and very low density lipoprotein synthesis is suggested by the findings that a significant fall in plasma triglyceride concentrations occurred in patients receiving both the 20 and $40 \mathrm{mg}$ twice daily doses of mevinolin. Such an effect was not observed at lower doses of mevinolin and it is tempting to speculate that the observed decreases in LDL on these doses $(5$ and $10 \mathrm{mg}$ twice daily) were mediated exclusively by increases in the rate of receptor-mediated LDL catabolism, whereas some concurrent reduction in LDL synthesis was apparent on the two higher doses.

The hypocholesterolemic effects of mevinolin (and compactin) are based on their ability to competitively inhibit hepatic HMG-COA reductase, the rate-limiting enzyme in cholesterol biosynthesis, and thereby reduce the rates of formation of mevalonic acid from HMG-COA and the de novo biosynthesis of cholesterol (32). The latter action is believed to transiently reduce the cellular cholesterol pool and result in compensatory increases in the number of high affinity LDL receptors on the cell surface (20) with a concurrent stimulation in the rate of receptor-mediated LDL catabolism $(20,29)$. This effect is similar to that which occurs in the liver when the entero-hepatic circulation of bile acids is interrupted either by oral administration of bile acid sequestrants, e.g., colestipol or cholestyramine $(20,21,33)$, or by surgical bypass of the distal ileum (15), but the mechanisms differ. In the former situation mevinolin directly reduces the rate of cholesterol biosynthesis, whereas in the latter case depletion of the bile salt pool promotes compensatory increases in the conversion of cholesterol to bile acids and indirectly increases the rate of cellular cholesterol efflux. In patients with heterozygous $\mathrm{FH}$, the ability of mevinolin and bile salt depletion to independently $(21,29)$ stimulate the production of more high affinity LDL receptors on hepatocytes affords a physiologically attractive and clinically effective means by which elevated LDL levels can be substantially reduced.

In addition to its role as a precursor of cholesterol, mevalonic acid is also an obligatory precursor (32) for the formation 
of ubiquinone (34), dolichol (35), and isopentenyl adenine (36). In view of the critical roles of these nonsterol products of mevalonate in cellular replication and function, it is essential that the doses of mevinolin that are used as hypolipidemic agents in man do not significantly impair the synthesis of ubiquinone, dolichols, or isopentenyl adenine. In vitro studies (reviewed in reference 32 ) indicate that the initial enzymes involved in the synthesis of ubiquinone and dolichol have a higher affinity for mevalonate and its metabolites than do the enzymes involved in cholesterol biosynthesis and that inhibition of HMG-COA reductase by compactin results in substantially greater reductions in the rates of synthesis of cholesterol than occur for dolichol or ubiquinone. Clinical data from seven patients with heterozygous FH treated with $30-60 \mathrm{mg} /$ day of compactin support this view (24). In this study, concentrations of total and LDL cholesterol fell by 32 and $29 \%$, respectively, but serum concentrations of ubiquinone were unchanged.

Short-term side effects during treatment with mevinolin have been minimal and all the patients reported in this study remain on therapy. Laboratory changes have been limited to transient increases in alkaline phosphatase in three patients and to a modest but sustained increase in a fourth. Previously reported side effects with mevinolin (27-29) and compactin (23-25) have also been minimal. Mabuchi et al. (24) reported an insignificant increase in the overall levels of CPK in seven Japanese patients treated with compactin at a dose of 30-60 $\mathrm{mg} / \mathrm{d}$, but similar changes were not reported in a subsequent study with 10 patients treated with $90 \mathrm{mg}$ of compactin/d (25). Tobert et al. (28) noted increased levels of CPK (two patients) and SGOT (two patients) in a group of 59 normal male subjects given mevinolin for a period of 1 mo. These results, along with those of the present study, indicate that mevinolin is well tolerated at clinically effective doses and that short-term side effects are minimal at doses of up to $1.5 \mathrm{mg} /$ kg per day. Nevertheless, until more information on the longterm safety of mevinolin in humans is available, it would seem prudent to restrict its use to selected adult patients with refractory hypercholesterolemia in whom the potential benefit from effective hypolipidemic therapy appears greatest.

If long-term safety can be satisfactorily established, specific inhibitors of HMG-COA reductase used alone or with either a bile acid sequestrant $(29,37)$, distal ileal bypass $(38)$, or nicotinic acid offer considerable promise in the therapy of patients with heterozygous FH and possibly in patients with other hereditary disorders (e.g., familial combined hyperlipidemia) that are associated with elevated levels of LDL cholesterol. The long-term use of such regimens affords the potential for preventing (39) or even reversing the premature development of atherosclerosis that occurs so frequently in this high risk group of patients.

\section{Acknowledgments}

We are grateful to Dr. J. A. Tobert (Merck Sharp \& Dohme Div., Merck \& Co., Inc., Rahway, NJ) for supplies of mevinolin; Anne
Kelleher; Ann Fanning, and the nursing and dietary staff of the Clinical Research Center for assistance; and to Mrs. Paula Bisaccio and Ms. Patricia Schade for preparation of this manuscript. We are also indebted to Dr. W. E. Connor for helpful discussions of this work, and to the patients involved in this study for their interest and cooperation.

This work was supported in part by National Institutes of Health research grants HL00953, HL29074, and HL32271, and by the General Clinical Research Centers Program (RR334).

Dr. Illingworth was the recipient of National Institutes of Health Research Career Development Award HL00953.

\section{References}

1. Jensen, J., D. H. Blankenhorn, and V. Kornerup. 1967. Coronary disease in familial hypercholesterolemia. Circulation. 36:77-82.

2. Slack, J. 1969. Risks of ischemic heart disease in familial hyperlipoproteinemic states. Lancet. II:1380-1382.

3. Stone, N. J., R. I. Levy, D. S. Fredrickson, and J. Verler. 1974. Coronary artery disease in 116 kindred with familial type II hyperlipoproteinemia. Circulation. 49:476-485.

4. Gagne, C., S. Moorjani, D. Brun, M. Toussaint, and P. J. Lupien. 1979. Heterozygous familial hypercholesterolemia: relationship between plasma lipids, lipoproteins, clinical manifestations, and ischemic heart disease in men and women. Atherosclerosis. 24:13-24.

5. Brown, M. S., and J. L. Goldstein. 1975. Familial hypercholesterolemia: genetic, biochemical, and pathological considerations. $A d v$. Intern. Med. 20:273-96.

6. Slack, J. 1979. Inheritance of familial hypercholesterolemia. Atheroscler. Rev. 5:35-66.

7. Goldstein, J. L., and M. S. Brown. 1983. Familial hypercholesterolemia. In The Metabolic Basis of Inherited Disease. J. B. Stanbury, J. B. Wyngaarden, D. S. Fredrickson, J. L. Goldstein, and M. S. Brown, editors. McGraw Hill, Inc., New York. Fifth ed. 672-712.

8. Langer, T., W. Strober, and R. I. Levy. 1972. The metabolism of low density lipoprotein in familial type II hyperlipoproteinemia. $J$. Clin. Invest. 51:1528-1536.

9. Bilheimer, D. W., N. J. Stone, and S. M. Grundy. 1979. Metabolic studies in familial hypercholesterolemia: evidence for a gene-dosage effect in vivo. J. Clin. Invest. 64:524-533.

10. Thompson, G. R., T. Spinks, A. Ranicar, and N. G. Myant. 1977. Non-steady state studies of low-density lipoprotein turnover in familial hypercholesterolemia. Clin. Sci. Mol. Med. 52:361-369.

11. Havel, R. J., and J. P. Kane. 1982. Therapy of hyperlipidemic states. Annu. Rev. Med. 33:417-433.

12. Cortese, C., C. B. Marenah, and B. Lewis. 1982. The effects of probucol on plasma lipoproteins in polygenic and familial hypercholesterolemia. Atherosclerosis. 44:319-325.

13. Illingworth, D. R., B. E. Phillipson, J. H. Rapp, and W. E. Connor. 1981. Colestipol plus nicotinic acid in treatment of heterozygous familial hypercholesterolemia. Lancet. I:296-298.

14. Kane, J. P., M. J. Malloy, P. Tun, N. R. Phillips, D. D. Freedman, M. L. Williams, J. S. Row, and R. J. Havel. 1981. Normalization of low density lipoprotein levels in heterozygous familial hypercholesterolemia with a combined drug regimen. N. Engl. J. Med. 304:251-258.

15. Spengel, F. A., A. Jadhav, R. G. M. Duffield, C. B. Wood, and G. R. Thompson. 1981. Superiority of partial ileal bypass over cholestyramine in reducing cholesterol in familial hypercholesterolemia. Lancet. II:768-770.

16. Levy, R. I., N. J. Stone, D. W. Bilheimer, W. V. Brown, D. S. 
Fredrickson, C. J. Glueck, A. M. Gotto, Jr., P. N. Herbert, P. O. Kwiterovich, T. Langer, J. Larosa, S. E. Lux, A. K. Rider, R. S. Shulman, and H. R. Sloan. 1973. Cholestyramine in type II hyperlipoproteinemia. Ann. Intern. Med. 79:41-58.

17. Glueck, C. J., S. Ford, D. Scheel, and P. Steiner. 1972. Colestipol and cholestyramine resin: comparative effects in familial type II hyperlipoproteinemia. JAMA. 222:675-680.

18. Lees, A. M., M. A. McCluskey, and R. S. Lees. 1976. Results of colestipol therapy in type II hyperlipoproteinemia. Atherosclerosis. 24:129-38.

19. Miettinen, T. A., and M. Lempinen. 1977. Cholestyramine and ileal bypass in the treatment of familial hypercholesterolemia. Eur. J. Clin. Invest. 7:509-514.

20. Kovanen, P. T., D. W. Bilheimer, J. L. Goldstein, and M. S. Brown. 1981. A regulatory role for hepatic low density lipoprotein receptors in vivo in the dog. Proc. Natl. Acad. Sci. USA. 78:11941198.

21. Shepherd, J., C. J. Packard, S. Becker, T. D. V. Lawrie, and H. G. Morgan. 1980. Cholestyramine promotes receptor-mediated low-density-lipoprotein catabolism. N. Engl. J. Med. 302:1219-1222.

22. Endo, A., M. Kuroda, and K. Tanzawa. 1976. Competitive inhibition of 3-hydroxy-3-methylglutaryl coenzyme A reductase by ML-236A and ML-236B, fungal metabolites having hypocholesterolemic activity. FEBS. Lett. 72:323-326.

23. Yamamoto, A., H. Sudo, and A. Endo. 1980. Therapeutic effects of ML236B in primary hypercholesterolemia. Atherosclerosis. 35:259-266.

24. Mabuchi, H., T. Haba, R. Tatami, S. Miyamoto, Y. Sakai, T. Wakasugi, A. Watanabe, J. Koizumi, and R. Takeda. 1981. Effects of an inhibitor of 3-hydroxy-3-methylglutaryl coenzyme A reductase on serum lipoproteins and ubiquinone-10 levels in patients with familial hypercholesterolemia. N. Engl. J. Med. 305:478-482.

25. Mabuchi, H., T. Sakai, Y. Sakai, A. Yoshimura, A. Watanabe, T. Wakasugi, J. Koizumi, and R. Takeda. 1983. Reduction of serum cholesterol in heterozygous patients with familial hypercholesterolemia: additive effects of compactin and cholestyramine. N. Engl. J. Med. 308:609-613.

26. Alberts, A. W., J. Chen, G. Kuron, V. Hunt, J. Huff, C. Hoffman, J. Rothrock, M. Lopez, H. Joshua, E. Harris, A. Patchett, R. Monaghan, S. Currie, E. Stapley, G. Albers-Schonberg, O. Hensens, J. Hirschfield, K. Hoogsteen, J. Liesch, and J. Springer. 1980. Mevinolin: a highly potent competitive inhibitor of 3-hydroxy 3-methyl glutaryl coenzyme A reductase and a cholesterol-lowering agent. Proc. Natl. Acad. Sci. USA. 77:3957-3961.

27. Tobert, J. A., G. Hitzenberger, W. R. Kutovetz, I. B. Holmes, and K. H. Jones. 1982. Rapid and substantial lowering of human serum cholesterol by mevinolin (MK803), an inhibitor of hydroxymethyl glutaryl coenzyme A reductase. Atherosclerosis. 41:61-65.

28. Tobert, J. A., G. D. Bell, J. Birtwell, I. James, W. R. Kukovetz, J. S. Pryor, A. Buntinx, I. B. Holmes, Y. S. Chao, and J. A. Bolognese. 1982. Cholesterol-lowering effect of mevinolin, an inhibitor of 3hydroxy-3-methylglutaryl coenzyme A reductase, in healthy volunteers. J. Clin. Invest. 69:913-919.

29. Bilheimer, D. W., S. M. Grundy, M. S. Brown, and J. L. Goldstein. 1983. Mevinolin and colestipol stimulate receptor-mediated clearance of low density lipoprotein from plasma in familial hypercholesterolemia heterozygotes. Proc. Natl. Acad. Sci. USA. 80:4124-4128.

30. National Heart, Lung, and Blood Institute. 1974. Lipid Research Clinics Manual 1. Dept. of Health, Education, and Welfare, National Institutes of Health, Bethesda, MD. Publication no. 75-628. 74.

31. Winer, B. J. 1971. Statistical Principles of Experimental Design. McGraw Hill Inc., New York. Second Ed. 261-273.

32. Brown, M. S., and J. L. Goldstein. 1980. Multivalent feedback regulation of HMG COA reductase, a control mechanism coordinating isoprenoid synthesis and cell growth. J. Lipid Res. 21:505-517.

33. Chao, Y. S., T. T. Yamin, and A. W. Alberts. 1982. Catabolism of low density lipoproteins by perfused rabbit livers: cholestyramine promotes receptor dependent hepatic catabolism of low density lipoproteins. Proc. Natl. Acad. Sci. USA. 79:3983-3986.

34. Trumpower, B. L. 1981. New concepts on the role of ubiquinone in the mitochondrial respiratory chain. J. Bioenerg. Biomembr. 13:124.

35. Mills, J. J., and A. M. Adamany. 1981. The role of phosphorylated dolichols in membrane glycoprotein synthesis: relation to cholesterol biosynthesis. Int. Rev. Cytol. 73:103-147.

36. Huneeus, V. Q., M. H. Wiley, and M. D. Siperstein. 1980. Isopentenyladenine as a mediator of mevalonate-regulated DNA replication. Proc. Natl. Acad. Sci. USA. 77:5842-5846.

37. Illingworth, D. R. 1983. Mevinolin in the therapy of heterozygous familial hypercholesterolemia. Circulation. 68:III-188. (Abstr.)

38. Illingworth, D. R., and W. E. Connor. 1984. Hypercholesterolemia persisting after distal ileal bypass: response to mevinolin. Ann. Intern. Med. 100:850-851.

39. Lipid Research Clinics Program. 1984. The lipid research clinics coronary primary prevention trial results. 1 . Reduction in incidence of coronary heart disease. JAMA. 251:351-364. 